\title{
NUCLEAR FRAGMENTATION
}

\author{
G. BERTSCH ${ }^{1}$ \\ Physics Department, Michigan State University, East Lansing, MI 48824, USA \\ and \\ Philip J. SIEMENS ${ }^{2}$ \\ Cyclotron Institute and Physics Department, Texas A\&M University, College Station, TX 77843, USA
}

Received 15 December 1982

Revised manuscript received 20 March 1983

\begin{abstract}
We consider the conditions under which nuclei will fragment into smaller pieces. We argue that fragmentation will occur when the nuclear matter has expanded adiabatically to the onset of hydrodynamic instability, $\partial P / \partial V / S=0$. We discuss the conditions of initial heating and/or compression which lead to fragmentation, and argue that the resulting fragmented matter will be composed mainly of alpha-particles and nucleons.
\end{abstract}

When enough energy is brought into a nucleus, it will break up into smaller pieces. This process is an important part of the cross section for reactions induced by high energy protons $[1,2]$. It is also seen in heavy ion collisions at projectile energies of the order of $20 \mathrm{MeV}$ per nucleon and higher $[3,4]$. However, there has been little theoretical work to date on the breakup process itself in the region of its threshold. Our present understanding of fragmentation is quite limited, being guided primarily by the numerical results of time-dependent Hartree-Fock theory.

In this note we analyze the bulk dynamics of nuclear matter under various conditions of density and internal energy, as a theoretical framework for describing the onset of fragmentation. We begin with the relation between the energy per particle of nuclear matter, $E$, the particle number density $n$, and the excitation energy per particle $I$,

$E=f(n)+I$.

1 Supported in part by Grant PHY-77-27084 from the US National Science Foundation.

2 Supported in part by Grant PHY-8109019 from the US National Science Foundation.
We call this the equation of state, although purists would reserve that designation for eq. (4) below. A typical example of an equation of state is given by the Skyrme parametrization,

$E=22.5\left(n / n_{0}\right)^{2 / 3}-62\left(n / n_{0}\right)^{2}+23.5\left(n / n_{0}\right)^{3}+I \mathrm{MeV}$

This is graphed in fig. 1. The parameters have been adjusted to reproduce the binding energy of nuclear matter, $E=-16 \mathrm{MeV}$ when $n=n_{0} \sim 0.16 / \mathrm{fm}^{3}$. The only additional information we need for the dynamics is the dependence of $E$ on $n$ at fixed entropy. In a non-interacting Fermi gas, the excitation energy behaves exactly the same way as does the total kinetic energy, i.e. it varies as the $2 / 3$ power of the density under isentropic changes in state.

$I(s, n)=I\left(s, n^{\prime}\right)\left(n / n^{\prime}\right)^{2 / 3}$.

In this respect, there is no difference between a Fermi gas and a classical Maxwell-Boltzmann gas of monoatomic particles. Some isentropes in the $(n, E)$ plane are shown as dashed lines in fig. 1. The Skyrme interaction would modify the excitation energy of eq. (3) with an extra factor $m / m^{*}$, where $m^{*}$ is the density. dependent effective mass. We suppose that $m \approx m^{*}$ 


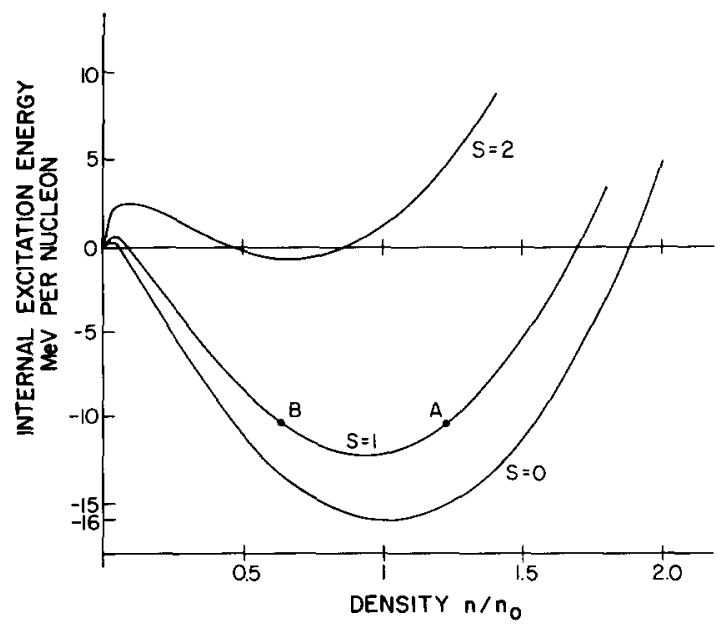

Fig. 1. Isentropes of nuclear matter in the $(n, E)$ plane [see text, eqs. (2) and (3)] .

from the density of states at the Fermi surface.

Nuclear matter will be in equilibrium if it is at an energy minimum of a isentrope. Otherwise, the pressure will be nonzero and the system will expand or contract. It is plausible to assume that the volume change takes place isentropically, in other words, that there is little dissipation associated with the expansion process. This result becomes exact both in the collisionless limit of mean-field theory, and when collisions are so frequent as to maintain local thermal equilibrium. Some evidence for this assumption is given by the existence of the giant monopole vibration, having a damping width much smaller than its frequency. Also, classical cascade calculations of high energy heavy ion collisions show not much entropy generation during the expansion phase of the collision [5] .

The pressure of the system is defined by

$P=n^{2} \partial E /\left.\partial n\right|_{S}$.

We now examine what happens to the system if we start it at some point in the $(n, E)$ plane with positive pressure. The system expands along the isentrope, converting internal energy $E$ into translational kinetic energy of the collective flow. For high enough initial energies, the pressure remains positive for all densities, and we may describe the process as an instantaneous vaporization. The pressure is always positive, starting from nuclear matter density, if the excitation energy is of the order of $20 \mathrm{MeV} / n$ or higher. The dynamics here will be featureless, with a relatively small fraction of composite particles in the final state.

At lower energies, the isentrope has a negative pressure portion. The expansion will be slowed down in this region, and will come to a halt when the kinetic energy of expansion has been converted back to internal energy. Thus the system will oscillate back and forth along the isentropes; starting from point $\mathrm{A}$ in fig. 1 it will reach point $B$ and no farther. This simple argument applies only if there are no phase transitions. If there is a separation between a condensed phase and a vapor phase, the thermodynamics becomes complicated. The usual gas-liquid phase transition is a first order transition tht a applies to processes that occur slowly enough for an equilibrium to be established across a phase boundary. As we envision it, the nuclear expansion process is rapid on the time scale required to establish such an equilibrium, so the usual gas-liquid equilibrium is not relevant to our considerations.

To achieve a phase separation on a short time scale, the system must develop a dynamic instability, i.e. an instability that will grow exponentially from a small amplitude. We will determine the dynamically unstable region by means of the compressibility,

$k=n \partial P /\left.\partial n\right|_{s}$.

The conventional nuclear physics parameter $K$ is related to $k$ by $K=9 k / n_{0}$. For our model equation of state, eq. (2), the compressibility is given by

$$
\begin{aligned}
& k / n_{0}=\frac{10}{9}\left(22.5+I_{0}\right)\left(n / n_{0}\right)^{5 / 3}-124\left(n / n_{0}\right)^{2} \\
& \quad+141\left(n / n_{0}\right)^{3} \mathrm{MeV} .
\end{aligned}
$$

Nuclear matter will be dynamically unstable when the compressibility is negative. The region of the $(n, E)$ plane in which this occurs is shown in fig. 2 . We believe that the occurrence of nuclear fragmentation, as a dominant reaction process, depends on whether the system enters into this unstable region. On the left nuclear matter will spontaneously form into droplets; on the right the mean field dynamics will preserve the homogeneous phase. Of course, quantum fluctuations will tend to smooth out sharp transitions in behavior, but the main processes should follow the mean field predictions.

Having defined the unstable region in the $(n, E)$ plane, we can ask how it is reached in a nuclear reac- 
tion. Initially, the reaction carries the system from the ground state to some point with higher internal energy. If the energy is transferred by a small object, there is not immediate change in the size of the system and therefore $n=n_{0}$. In a heavy collision, the interpenetrating nuclei create a state of higher density, $n>n_{0}$. From this excited position, the system will then expand along an isentrope until a point of equal internal energy is reached on the left. In fig. 2, we have mapped out the region of starting conditions which will reach the instability upon expansion. If the initial condition is to the right of the dashed line, the system has enough energy to expand into the unstable region.

An example of a reaction process which does not change the initial density very much is the fragmentation induced by ultrarelativistic protons. Much of the fragment production can be explained by evaporation of the target [6], but part of the cross section is thought to be due to a fast fragmentation process [7, 8]. The typical energy deposited is of the order of $1-$ $2 \mathrm{GeV}$. Thus, for $A=100$, the system would have an energy about $10 \mathrm{MeV} / n$, which is just at the threshold of the $n=n_{0}$ instability region. The precise location of the threshold depends on the details of the equation of state; an equation of state with compressibility consistent with the monopole vibration frequency, $K$ $=190 \mathrm{MeV}$, has a threshold at $I=8.5 \mathrm{MeV}$.

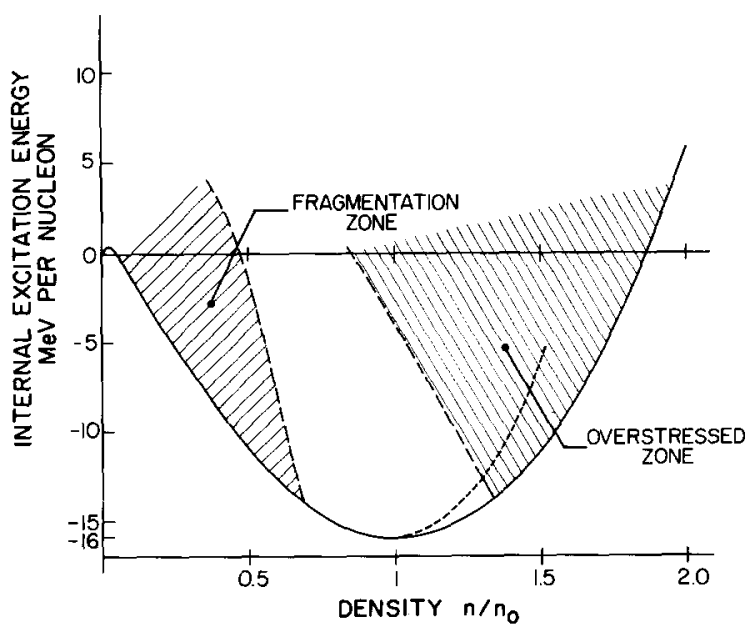

Fig. 2. Instabilities of nuclear matter. Left grating: region of hydrodynamic instability, $(\partial P / \partial V)_{S}=0$. Right grating: initial conditions leading to breakup after expansion. Dashed line: hydrodynamic compression, eq. (8).
In heavy ion collisions, bulk dynamics predicts that the available energy and the density compression have a quadratic relationship,

$E=a\left(n-n_{0}\right)^{2}$,

with the coefficient $a$ depending on the equation of state. The stiffer the equation of state, the closer eq. (8) will be to the $s=0$ isentrope. The one-dimensional TDHF theory with an equation of state of the type eq. (2) predicts a compression to $1.4 n_{0}$ with an energy of $10 \mathrm{MeV} / n$ [9]. The curve of eq. (8) going through this point is marked in fig. 2. Assuming that all of the excitation energy was thermalized, this would give a threshold for fragmentation at $3.5 \mathrm{MeV} / n$. Of course, in TDHF, the excitation energy is in a coherent deformation of the Fermi surface, and under those circumstances the bulk dynamics predicts a lower threshold [10]. In any case, there is some rather low threshold, and above this energy, the system always comes apart.

If the unstable matter extends over a large spatial region, the formation of droplets will favor those sizes which have wavelengths corresponding to the maximum growth rate. This could be calculated from the (imaginary) frequencies of the RPA modes; however, since frequencies increase for shorter wavelengths, we anticipate that the Fermi momentum would set the scale, and that alpha-particle sized drops would be most favored. A similar conclusion is reached if we assume that the instabilities grow chaotically, leading to a state of maximum entropy in which alpha particles will also be the predominant fragments.

We identify the instability of the bulk dynamics with the "fusion window" predicted in TDHF [11]. For energies well above the fusion window, we expect that heavy ion collisions will produce many alphaparticles in the central region. These should not be difficult to distinguish from evaporation alpha-particles, and would give useful information on the equation of state and the thermalization in the early stages of the collision. In particular, the onset of fragmentation can be used to help infer the density at which thermalization takes place: according to fig. 2 , the energy threshold for fragmentation is an increasing function of the initial density.

We would like to thank H. Toki and J.P. Bondorf for discussions. 


\section{References}

[1] G.D. Westfall et al., Phys. Rev. C17 (1978) 1368.

[2] J.E. Finn et al., Purdue University preprint (1982).

[3] C. Egelhaaf et al., Phys. Rev. Lett. 46 (1981) 318.

[4] Y. Viyogi et al., Phys. Rev. Lett. 42 (1979) 33.

[5] G. Bertsch and J. Cugnon, Phys. Rev. C24 (1981) 2514.

[6] X. Campi and J. Huefner, Phys. Rev. C24 (1981) 2199.
[7] S. Bohrmann, J. Huefner and M. Nemes, Heidelberg preprint, MPIH-V16 (1982).

[8] H. Araseki and T. Fujita, Nihon University preprint (1982).

[9] P. Bonche, S. Koonin and J. Negele, Phys. Rev. C13 (1976) 1226.

[10] G. Bertsch and G. Mundiger, Phys. Rev. C17 (1978) 1646.

[11] H. Flocard and M.S. Weiss, Phys. Rev. C18 (1978) 573. 